\title{
Atelocollagen-mediated in vivo siRNA transfection in ovarian carcinoma is influenced by tumor site, siRNA target and administration route
}

\author{
MATTHIEU MERYET-FIGUIÈRE ${ }^{1-3 *}$, CHARLOTTE LECERF $^{1-3 *}$, EMILIE VARIN $^{1-3^{*}}$, JEAN-LUC COLL $^{5}$, \\ MARIE-HÉLÈNE LOUIS ${ }^{1-3}$, SOIZIC DUTOIT ${ }^{1-3}$, FLORENCE GIFFARD ${ }^{1-3}$, CÉCILE BLANC-FOURNIER ${ }^{1-4}$, \\ SIHAM HEDIR $^{1-3}$, NICOLAS VIGNERON ${ }^{1-3}$, EMILIE BROTIN ${ }^{1-3}$, LAURENT PELLETIER $^{6}$, \\ VÉRONIQUE JOSSERAND ${ }^{5}$, CHRISTOPHE DENOYELLE ${ }^{1-3}$ and LAURENT POULAIN ${ }^{1-3}$ \\ ${ }^{1}$ INSERM U1086 ‘ANTICIPE’ Interdisciplinary Research Unit for Cancer Prevention and Treatment, \\ Axe 2: 'Biology and Innovative Therapeutics for Locally Aggressive Cancers' (BioTICLA), \\ Comprehensive Cancer Center François Baclesse, 14076 Caen Cedex $5 ;{ }^{2}$ Normandie Univ, Normandie Université, \\ Esplanade de la Paix, 14032 Caen Cedex 5; ${ }^{3}$ Comprehensive Cancer Centre F. Baclesse, Unicancer, 14076 Caen Cedex 5; \\ ${ }^{4}$ Laboratory of Pathology, Comprehensive Cancer Centre François Baclesse, Unicancer, 14076 Caen Cedex 5; \\ ${ }^{5}$ INSERM U1209, Institute of Advanced Biosciences, Institut pour l'Avancée des Biosciences, Centre de Recherche UGA, \\ Site Santé, 38700 La Tronche; ${ }^{6}$ INSERM U836, Grenoble Institute of Neurosciences, Bâtiment Edmond J. Safra, \\ Chemin Fortuné Ferrini, Site Santé, 38706 La Tronche Cedex, France
}

Received February 16, 2017; Accepted June 12, 2017

DOI: 10.3892/or.2017.5882

\begin{abstract}
Ovarian cancer is the leading cause of death from gynecological malignancies worldwide, and innate or acquired chemoresistance of ovarian cancer cells is the major cause of therapeutic failure. It has been demonstrated that the concomitant inhibition of Bcl- $\mathrm{x}_{\mathrm{L}}$ and Mcl-1 anti-apoptotic activities is able to trigger apoptosis in chemoresistant ovarian cancer cells. In this context, siRNA-mediated $\mathrm{Bcl}-\mathrm{x}_{\mathrm{L}}$ and Mcl-1 inhibition constitutes an appealing strategy by which to eliminate chemoresistant cancer cells. However, the safest and most efficient way to vectorize siRNAs in vivo is still under debate. In the present study, using in vivo bioluminescence imaging, we evaluated the interest of atelocollagen to
\end{abstract}

Correspondence to: Dr Matthieu Meryet-Figuière, INSERM U1086 'ANTICIPE' Interdisciplinary Research Unit for Cancer Prevention and Treatment, Axe 2: 'Biology and Innovative Therapeutics for Locally Aggressive Cancers' (BioTICLA), Comprehensive Cancer Center François Baclesse, 3 Avenue du Général Harris, BP 5026, 14076 Caen Cedex 5, France

E-mail: m.meryet-figuiere@baclesse.unicancer.fr

${ }^{*}$ Contributed equally

Abbreviations: HES, hematoxylin, eosin, safran; s.c., subcutaneous; i.p., intra-peritoneal; i.v., intravenous; ASO, antisense oligonucleotide

Key words: ovarian cancer, in vivo transfection, siRNA, atelocollagen, Bcl-2 family vectorize siRNAs by intraperitoneal (i.p.) or intravenous (i.v.) administration in 2 xenografted ovarian cancer models (peritoneal carcinomatosis and subcutaneous tumors in nude mice). Whereas i.p. administration of atelocollagen-vectorized siRNA in the peritoneal carcinomatosis model did not induce any gene downregulation, a $70 \%$ transient downregulation of luciferase expression was achieved after i.v. injection of atelocollagen-vectorized siRNA in the subcutaneous (s.c.) model. However, the use of siRNA targeting Bcl- $\mathrm{x}_{\mathrm{L}}$ or Mcl-1 did not induce target-specific downregulation in vivo in nude mice. Our results therefore show that atelocollagen complex formulation, the administration route, tumor site and the identity of the siRNA target influence the efficiency of atelocollagen-mediated siRNA delivery.

\section{Introduction}

Ovarian carcinoma therapeutic care remains inefficient owing to the high level of chemoresistance to conventional treatment. With approximately 145,000 new cases each year in developed countries and approximately 100,000 deaths, ovarian carcinoma is the first leading cause of death from gynecological malignancies.

We previously showed that apoptosis control defects are responsible for the strong protection of ovarian cancer cells against cell death $(1,2)$. In particular, we demonstrated that the concomitant inhibition of the anti-apoptotic proteins Bcl- $\mathrm{x}_{\mathrm{L}}$ and Mcl-1 either by siRNAs $(3,4)$, or by combining a BH3-mimetic molecule targeting Bcl- $\mathrm{x}_{\mathrm{L}}$ with a siRNA or a BH3-mimetic molecule targeting Mcl-1 (5-8) triggers massive apoptotic cell death in chemoresistant ovarian cancer cells in vitro, even in the absence of chemotherapeutic treatment. 
This effect is due to the 'primed for death' feature of cancer cells (9). Unlike normal cells, cancer cells contain a high level of the activated apoptosis inducers Bax and Bak, or activator BH3-only proteins such as Bim and Puma, which are sequestered by anti-apoptotic proteins such as Bcl- $\mathrm{x}_{\mathrm{L}}$ or Mcl-1 (10). Strategies aimed at the release of Bax/Bak or Bim/Puma from their inhibitors are thus highly attractive in a clinical perspective. In this context, the design of innovative BH3-mimetic molecules able to target $\mathrm{Bcl}-\mathrm{x}_{\mathrm{L}}$ in vivo, such as ABT-263 (Navitoclax), opens up new perspectives for clinical validation of such approaches. However, pharmacological inhibition of Mcl-1 in vivo remains problematic despite recent promising advances $(8,11-13)$.

The versatility in the choice of siRNA targets as well as their high efficiency for the downregulation of a specific gene makes the perspective of clinically available siRNA strategies highly attractive. Recent advances in chemistry are now allowing a panel of chemical modifications in siRNAs that avoid unwanted immunostimulatory and off-target effects, thus overcoming some of the major issues preventing the safe and efficient use of siRNAs in clinical practice (14). However, efficient vectorization and delivery of siRNAs in vivo remains a major challenge (15), despite the huge amount of research carried out on this topic.

Among the numerous strategies developed for the in vivo vectorization of siRNAs (16), atelocollagen constitutes an interesting option. Atelocollagen is a derivative of collagen that has been used for the in vivo transfection of nucleic acids such as DNA, antisense oligonucleotides (ASO) and siRNA (17). It is processed from bovine dermal collagen after digestion of pepsine-mediated telopeptides to avoid immunogenicity (18). Atelocollagen is a highly biocompatible and biodegradable compound used in numerous medicinal, surgical and cosmetic applications.

A molecular model has been proposed for atelocollagen/siRNA interactions in which the interaction between atelocollagen, which is positively charged in physiological conditions (19), and the siRNA phosphates promotes the formation of a fibrillar structure containing 5 atelocollagen molecules for 1 siRNA duplex (20). This structure efficiently protects the siRNA from nucleases and is compatible with cellular uptake. In addition, modification of the atelocollagen concentration during complex formation modifies the viscosity of the final product. Low concentration formulations lead to a viscosity close to that of blood and are adapted to intravenous administration. Conversely, higher atelocollagen concentrations lead to a gel-like formation, allowing the continuous delivery of siRNA over an extended period of time after local administration. This plasticity of formulation enables the use of atelocollagen for various administration routes (intravenous, intraperitoneal, intramuscular, intratumoral, peritumoral) $(19,21)$. Atelocollagen has thus been proposed as an adequate vector for the in vivo local or systemic siRNA administration in various tumor models (17,21-24).

Several studies exploring the antitumor effects of atelocollagen-mediated siRNA administration have utilized either intratumoral (24-29) or peritumoral $(18,22,30-32)$ administration. The only study describing atelocollagen delivery of siRNA in a subcutaneous ovarian cancer model used peritumoral administration (32). Using a vasohibin 2 siRNA to target angiogenesis, the authors reported a decrease in tumor growth, tumor dissemination and angiogenesis. However, intratumoral or peritumoral administration is not suitable for treating ovarian carcinoma, in which peritoneal carcinomatosis is sometimes composed of hundreds of disseminated tumor nodes.

The poor overall survival of ovarian carcinoma patients is mainly due to disease recurrence within the peritoneal cavity (33). We thus decided to explore the possibility of siRNA delivery in peritoneal carcinomatosis. In this context, intraperitoneal (i.p.) administration is an attractive option, particularly since the small tumor nodes lack vascularization. Furthermore, local treatment carries a reduced risk of systemic toxicity and could retain some of the properties of peritumoral injections, at least next to the injection site and possibly throughout the peritoneal compartment. Atelocollagen delivery of siRNA via the i.p. route has been used in several studies $(33,34)$, providing an interesting proof-of-concept.

Numerous studies with in vivo administration of siRNA-vectorized by atelocollagen involve intravenous (i.v.) administration $(24,35,36)$. It is more feasible in routine clinical use due to the reduced risk for patients compared to i.p. administration as the use of catheters for repeated treatments can lead to serious side-effects (34). Moreover, i.v.-delivered particles likely reach 'in-depth' larger vascularized tumor nodes more easily. The opportunity to deliver siRNAs via systemic administration would make it suitable for several pathologies, including cancer, provided that the chosen site of delivery is vascularized. In the context of ovarian carcinoma, this would mean that peritoneal tumor nodes of only a few millimeters in size would be amenable to siRNA delivery.

As both i.p. and i.v. routes present significant advantages in the context of ovarian carcinoma, we decided to explore the efficiency of atelocollagen-vectorized siRNA delivery in two independent models: a peritoneal carcinomatosis model submitted to i.p. siRNA/vector complex injection, and an s.c. vascularized tumor node model submitted to i.v. siRNA/vector complex injection. To this end, we first used longitudinal imaging studies performed on luciferase-expressing SKOV3 tumors to define optimal administration modalities in both models. Our results evidenced the interest of i.v. administration in an s.c. model of tumor development. We next studied these administration modalities for the siRNA-mediated silencing of Bcl- $\mathrm{x}_{\mathrm{L}}$ and $\mathrm{Mcl}-1$ in the same animal models.

\section{Materials and methods}

Cell culture and treatment. SKOV3 ovarian carcinoma cell line and its luciferase-expressing counterpart SKOV3-Luc were obtained from the American Type Culture Collection (ATCC; Manassas, VA, USA). They were grown in RPMI-1640 medium supplemented with $2 \mathrm{mM}$ Glutamax $^{\mathrm{TM}}, 25 \mu \mathrm{M}$ HEPES, $10 \%$ fetal calf serum and $33 \mathrm{mM}$ sodium bicarbonate (Fisher Scientific Bioblock, Illkirch, France) and were maintained in a $5 \% \mathrm{CO}_{2}$ humidified atmosphere at $37^{\circ} \mathrm{C}$.

In vitro transfection. All siRNAs used in the present study were chemically synthesized and PAGE-purified by Eurogentec (Liège, Belgium) and received as annealed oligonucleotides. siRNA guide sequences targeting the 
indicated genes were as follows: Mcl-1 siRNA (noted siMcl1), 5'-uguuuagccacaaaggcac-3', Bcl- $\mathrm{X}_{\mathrm{L}}$ siRNA (noted siBcl-xL), 5'-ugcgauccgacucaccaau-3', luciferase siRNA (noted siLuc), 5'-ucgaaguacucagcguaag-3' and GFP siRNA (noted siGFP, negative control), 5'-acuuguggccguuuacguc-3'. Exponentially growing cells were seeded $\left(2.5 \times 10^{5}\right.$ cells $/ 25 \mathrm{~cm}^{2}$ flask $)$ the day before transfection to reach $30-50 \%$ confluency at the time of transfection. Briefly, the transfecting reagent INTERFERin $^{\mathrm{TM}}$ (Polyplus Transfection, Strasbourg, France) was added to siRNA diluted in OptiMEM ${ }^{\circledR}$ serum-free medium (Life Technologies, Saint Aubin, France), and the formation of siRNA/vector complexes was allowed to proceed for $15 \mathrm{~min}$ at RT before distribution in culture flasks. The next day, cell medium was changed to remove the remaining transfecting reagent. At the indicated time, cells were trypsinized and washed with cold phosphate-buffered saline (PBS). Cell pellets were used directly or stored at $-80^{\circ} \mathrm{C}$ for later use.

In vitro bioluminescence measurement. Firefly luciferase activity was assayed $72 \mathrm{~h}$ after siLuc transfection with the Dual-Luciferase Reporter Assay System (Promega) and measured with a luminometer Centro LB 960 (Berthold, Thoiry, France). Each assay contained 3 technical replicates.

Animal experiments. Female nude mice (Janvier Laboratories, Saint Berthevin, France) 6-weeks of age were injected with human ovarian carcinoma cell lines (SKOV3-Luc or SKOV3) i.p. $\left(20 \times 10^{6}\right.$ cells in $200 \mu \mathrm{l}$ PBS $)$ or s.c. bilaterally on the flanks $\left(5 \times 10^{6}\right.$ cells in $200 \mu \mathrm{l}$ PBS). The animals were kept under pathogen-free conditions and fed and watered ad libitum, in cages of 4 to 5 animals (in compliance with recommended area surface/animal), in a dedicated room with a $12 \mathrm{~h} / 12 \mathrm{~h}$ light/dark cycle at a constant temperature of $22^{\circ} \mathrm{C}$. A period of 4 weeks was allowed for tumor development before the beginning of the experiments. In vivo experiments were performed in the animal core facilities of the Comprehensive Cancer Centre F. Baclesse in Caen and of the Institute of Advanced Biosciences in Grenoble, both of which are certified by the French Ministry of Higher Education and Research and regularly controlled by the Departmental Management of Population Protection (certification nos. B14 118003 and C38 516 10001, respectively). Animal experiments were performed according to the current regulations in the respect of animal experiment ethics. Animals were euthanatized at the end points of the experiments, i.e. after a total of 5 weeks of tumoral development. At this stage, s.c. tumors were $\sim 4 \mathrm{~mm}$ in size, $33.5 \mathrm{~mm}^{3}$ ( $\left.\mathrm{V}=\mathrm{L} \times 12 \times \pi / 6\right)$, whereas for micro-carcinomatosis, nodules were just becoming visible on the peritoneum with the presence of a few nodules (usually 1-5) $\sim 10 \mathrm{~mm}^{3}$ in size in the peritoneal cavity. At this stage no ascites was noted. For euthanasia, animals were anesthetized with a gaseous mix of isoflurane/oxygen (5/95) and thereafter euthanatized with a gaseous mix of oxygen/carbon dioxide (20/80).

In vivo treatments. For local administration, $100 \mu \mathrm{g}$ of siRNA complexed with atelocollagen at a final $0.5 \%$ concentration was injected i.p. in $400 \mu \mathrm{l}$. For systemic administration, $125 \mu \mathrm{g}$ of siRNA complexed with atelocollagen at a final concentration of $0.05 \%$ was injected i.v. in $200 \mu \mathrm{l}$. $\mathrm{NaCl}$ injections for control animals were performed with the same volumes.
Local ( $0.5 \%$ Atelocollagen) or systemic ( $0.05 \%$ Atelocollagen) administration kits were purchased from Cosmobio (Koken Co., Ltd., Tokyo, Japan). siRNA/vector complexes were prepared according to the manufacturer's protocol for local use kit, and with a modified protocol, i.e. increase in siRNA concentration in the formulation for the systemic kit, according to the protocol published by $\mathrm{Mu}$ et al (24). In experiments involving siMcl1 and siBcl- $\mathrm{x}_{\mathrm{L}}$, animals were distributed into 3 subgroups prior to treatment according to the size of the s.c. tumor, i.e. large, medium and small. We thus avoided any possible bias due to unequal average tumor size between groups. Mice from these subgroups were then equally distributed into 4 experimental groups of 5 mice each for a total of 20 mice: vehicle ( $\mathrm{NaCl}$ ), control siRNA (siGFP), Mcl-1 siRNA (siMcl1) and Bcl- $x_{\mathrm{L}}$ siRNA $\left(\operatorname{siBcl}-\mathrm{x}_{\mathrm{L}}\right)$.

Bioluminescence imaging. In vivo imaging was conducted at Optimal (Grenoble, France), a core facility for small animal optical imaging. After 4 weeks of tumor development, mice received an i.p. injection of $150 \mu \mathrm{g} / \mathrm{g}$ luciferin (Promega, Charbonnières-les-Bains, France) for non-invasive bioluminescence imaging (IVIS Kinetic; PerkinElmer, Waltham, MA, USA). Semi-quantitative data of luciferase-positive tumor cell signals were obtained using the manufacturer's software (Living Image; PerkinElmer). Results were expressed as photons/second (photons/s). For animal randomization prior to treatment, mice were separated into 3 subgroups with high, medium and low levels of bioluminescent signals. Mice from each of these subgroups were then distributed into 3 experimental groups for a total of 20 mice: vehicle $(\mathrm{NaCl}$, 4 and 3 mice bearing i.p. or s.c. tumors respectively), control siRNA (siGFP, 3 and 3 mice bearing i.p. or s.c. tumors, respectively) and luciferase siRNA (siLuc, 4 and 3 mice bearing i.p. or s.c. tumors, respectively) treatment. Bioluminescence imaging was performed at day 0,3 and 7. Results were then expressed as values relative to day 0 .

Statistical analysis. In vivo luciferase assays were compared using the non-parametrical Wilcoxon rank-sum test, and p-values $<0.05$ were considered as significant. Statistical analyses were calculated using the 32-bit R Console software V3.1.0 (R Foundation for Statistical Computing, Vienna, Austria).

Immunohistochemical analysis. Automated immunohistochemistry using a Ventana Discovery XT autostainer was performed on $4 \mu \mathrm{m}$-thick paraffin sections. Slides were deparaffinized with EZPrep buffer and epitopes were unmasked by $15 \mathrm{~min}$ of high-temperature treatment in CC1 EDTA buffer. Sections were incubated for $40 \mathrm{~min}$ at $37^{\circ} \mathrm{C}$ with an anti Mcl-1 (ab32087; Abcam, Cambridge, MA, USA) or Bcl- $\mathrm{x}_{\mathrm{L}}$ antibody (556361; BD Pharmingen, Franklin Lakes, NJ, USA). Secondary antibody (Omnimap Rabbit; Ventana Medical System Inc., Tucson, AZ, USA) was incubated for $16 \mathrm{~min}$ at room temperature. Immunodetection performed without the primary antibody was used as the control. After washes, the staining was performed with DAB and sections were counterstained with hematoxylin using Ventana reagents according to the manufacturer's protocol. Stained slides were then digitized using an Aperio ScanScope slide scanner (Aperio Technologies, Vista, CA, USA). 

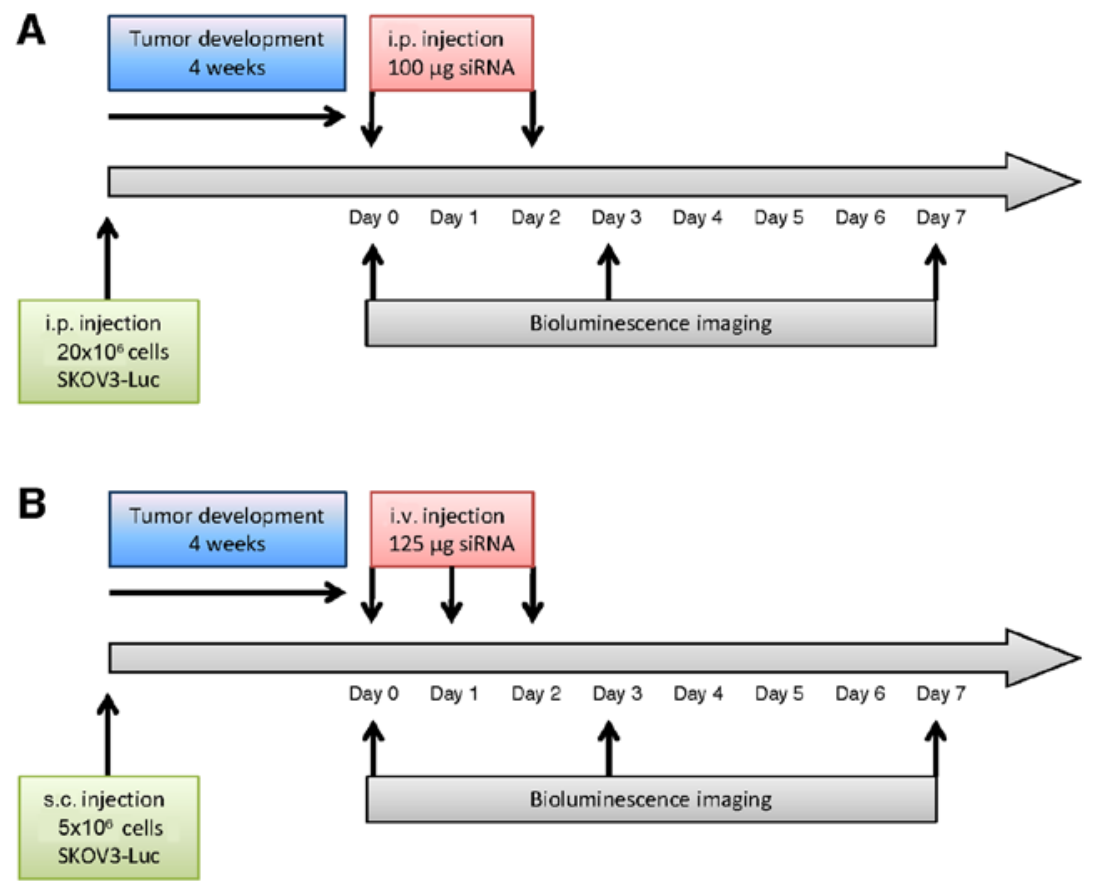

Figure 1. Schedules for in vivo siRNA treatments. (A) Mice were injected i.p. with 20x10 ${ }^{6}$ SKOV3-Luc cells and tumors were allowed to develop for 4 weeks. Bioluminescence values were acquired at day 0 prior to treatment, day 3 and 7. Atelocollagen ( $0.5 \%$ final) in complex with $100 \mu \mathrm{g}$ siRNA was injected i.p. at day 0 and 2. (B) Mice were injected bilaterally s.c. with $5 \times 10^{6}$ SKOV3-Luc cells in each flank and tumors were allowed to develop for 4 weeks. Bioluminescence values were acquired at day 0 prior to treatment, day 3 and 7 . Atelocollagen $(0.05 \%$ final) in complex with $125 \mu \mathrm{g}$ siRNA was injected i.p. at days 0,1 and 2 .

A

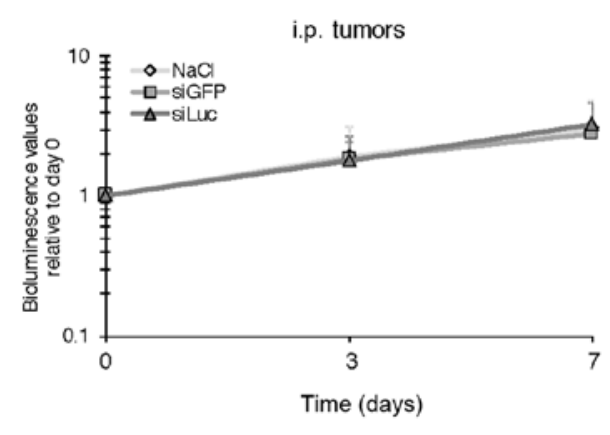

C

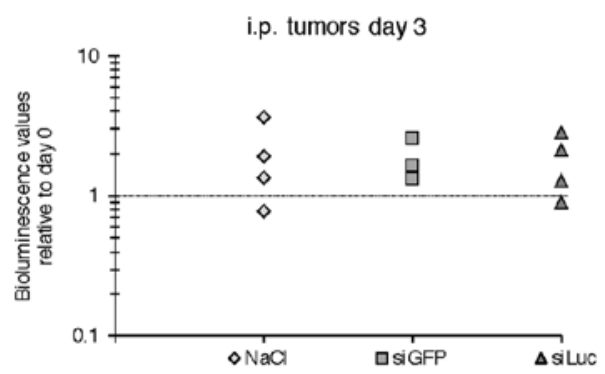

B
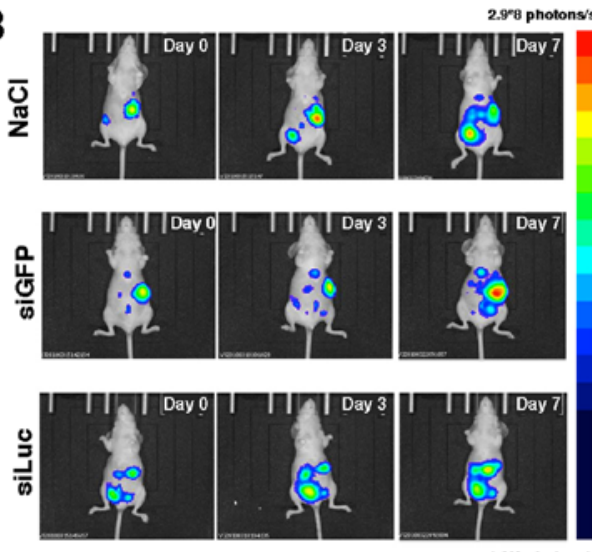

D

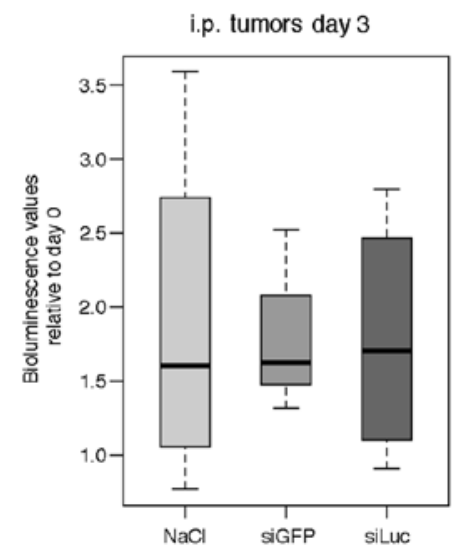

Figure 2. i.p. injection for luciferase-targeted siRNA to i.p. carcinomatosis. (A) Evolution of the bioluminescent signal over time for NaCl- (4 mice), siGFP- (3 mice) and siLuc- (4 mice)-treated groups. Mean values \pm SD for each group of animals relative to bioluminescence values acquired at day 0 for each individual. (B) Representative bioluminescence image for one animal from each group. (C) Bioluminescence values at day 3 for individual mice from each group relative to bioluminescence values acquired at day 0. (D) Box plot of bioluminescence values of each group of animals measured at day 3 relative to bioluminescence values acquired at day 0 . No significant difference between groups. 
A

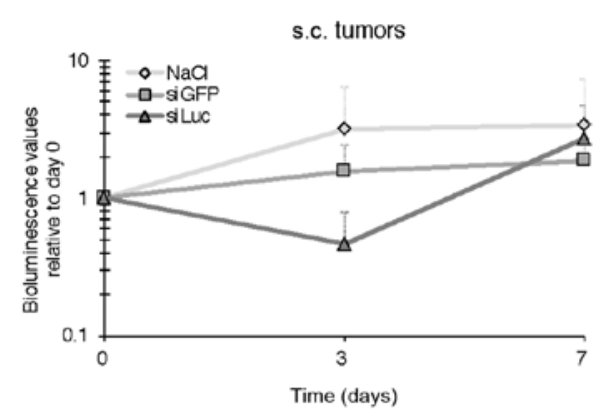

C

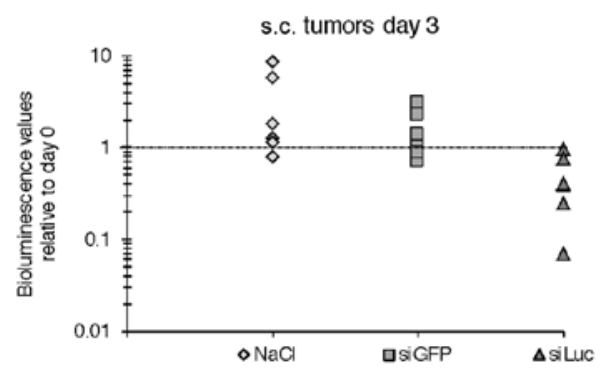

B
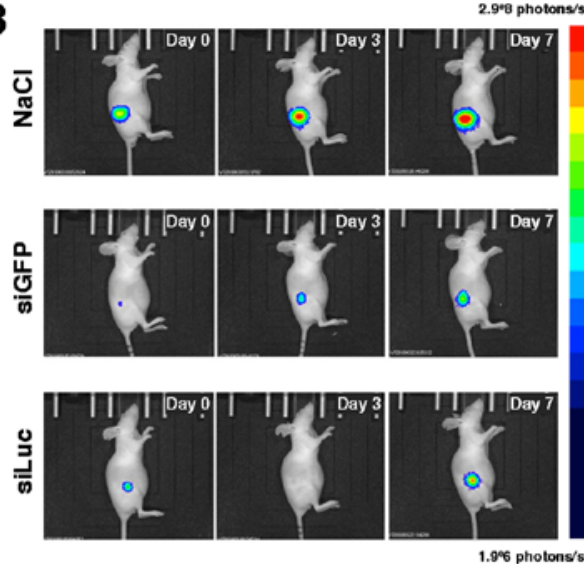

D

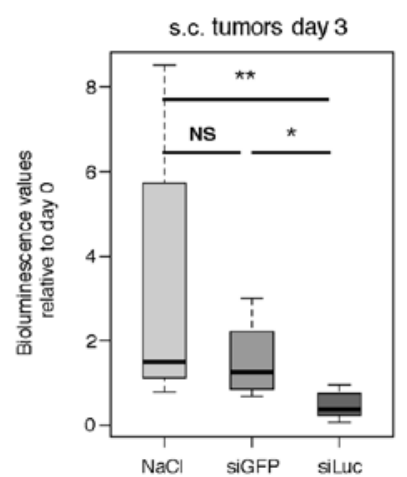

Figure 3. i.v. injection for luciferase-targeted siRNA to subcutaneous tumors. (A) Evolution of the bioluminescence signal over time for NaCl- (3 mice), siGFP- (3 mice) and siLuc (3 mice)-treated groups. Mean values \pm SD for tumors from each group of animals relative to bioluminescence values acquired at day 0 for each tumor. (B) Representative bioluminescence image for one animal from each group. (C) Bioluminescence values at day 3 for individual tumors from each group relative to bioluminescence values acquired at day 0. (D) Box plot of bioluminescence values of each group of animals measured at day 3 relative to bioluminescence values acquired at day 0 . The siLuc-treated group was significantly different from the $\mathrm{NaCl}-(\mathrm{p}=0.004)$ and siGFP $(\mathrm{p}=0.015)$-treated groups (Wilcoxon rank-sum test).

$R N A$ isolation and quantitative reverse transcriptase-PCR $(R T-P C R)$. Total RNA was isolated from the SKOV3 cell line using TRIzol (Qiagen, Courtaboeuf, France) and $1 \mu \mathrm{g}$ was reverse-transcribed using Omniscript reverse transcriptase (Qiagen) with random hexamers according to the manufacturer's instructions. cDNA was combined with $10 \mu \mathrm{mol} / 1$ of each forward and reverse primer, $50 \mu \mathrm{mol} / 1$ of the TaqMan ${ }^{\circledR}$ probe and 2X TaqMan ${ }^{\circledR}$ Fast Universal PCR Master Mix (Applied Biosystems, Foster City, CA, USA) in a $20 \mu \mathrm{l}$ final reaction volume. The following probes were used: for Mcl-1

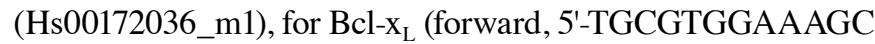
GTAGACAA-3' and reverse, 5'-AGGTAAGTGGCCATCCAA GCT-3'; probe, 5'-AGATGCAGGTATTGGTG-3') and GAPDH (Hs99999905_m1). All PCR amplification reactions were carried out in triplicate on an Applied ABI Prism 7500 Fast PCR system (Applied Biosystems). The Mcl-1 and Bcl- $\mathrm{x}_{\mathrm{L}}$ transcripts were quantified relative to GAPDH endogenous reference by the comparative $2^{-\Delta \Delta \mathrm{Ct}}$ method and results are expressed as a percentage of untransfected tumors from $\mathrm{NaCl}$-injected mice.

\section{Results}

Nude mice were xenografted with SKOV3 ovarian cancer cells constitutively expressing luciferase (SKOV3-Luc) by i.p. or bilateral s.c. injection to induce the development of peritoneal carcinomatosis or s.c. tumors, respectively. The use of in vivo bioluminescence imaging allowed individual longitudinal follow-up of tumor development for each animal. Therefore, by measuring the evolution of bioluminescence signals over time, each tumor constitutes its own control, thus limiting the heterogeneity of response due to inter-animal variation in the rate of tumor development.

Atelocollagen-vectorized siRNA targeting luciferase injected by i.p. route does not decrease bioluminescence in carcinomatosis tumor nodes. In the peritoneal carcinomatosis model, atelocollagen was complexed with siRNA to a 'high' final concentration of atelocollagen $(0.5 \%)$ to obtain a gel-like compound, according to the manufacturer's protocol. This gel-like formulation was used to allow the continuous release of siRNA in the peritoneal cavity. siRNA/vector complexes or vehicle were injected i.p. at day 0 and day 2 . Bioluminescence was measured at day 0 (before treatment), day 3 and day 7 . Treatment and the imaging schedules are represented in Fig. 1A.

As expected, an increase in the bioluminescence signal was detected over time from day 0 to 3 , and day 7 in the control tumors, corresponding to tumor growth. However, siLuc-treated animals did not display any decrease in bioluminescent signal (178 and $325 \%$ of the signal at day 3 and day 7 , respectively, relative to day 0) compared to siGFP (182 and $278 \%$ ) or vehicle (189 and 302\%) (Fig. 2 and data not shown). 


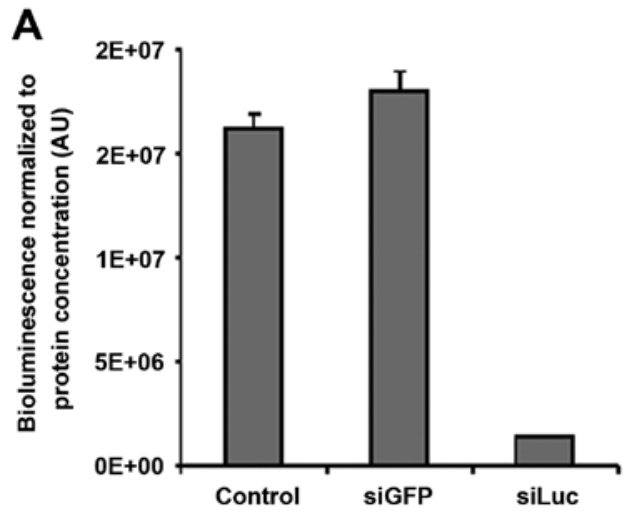

B

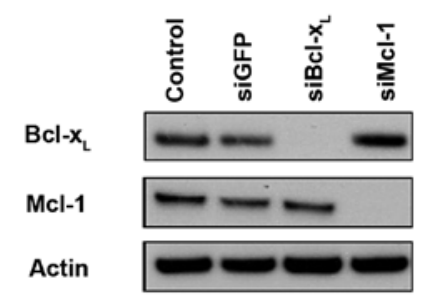

Figure 4. In vitro efficiency of the siRNA batches used in vivo. (A) Luciferase-targeted siRNAs strongly decrease target expression in vitro. Transfection was performed with siLuc in SKOV3-Luc cells with final $20 \mathrm{nM}$ of siRNAs, and target downregulation analyzed $72 \mathrm{~h}$ after transfection. Bioluminescent activity was measured in cell lysates. Results are relative to protein concentration in respective cell lysates $\pm \mathrm{SD}$. (B) Both Bcl- $\mathrm{x}_{\mathrm{L}}$ - and Mcl-1-targeted siRNAs mediate strong downregulation in vitro on their respective targets. Transfection was performed with siGFP, siMcll or siBcl- $\mathrm{x}_{\mathrm{L}}$ in SKOV3 cells with final $20 \mathrm{nM}$ of siRNAs, and target downregulation analyzed $72 \mathrm{~h}$ after transfection. Mcl-1 and Bcl- $\mathrm{x}_{\mathrm{L}}$ protein expression was assessed by western blotting. Actin was used as a loading control.

Atelocollagen-vectorized siRNA targeting luciferase injected i.v. decreases bioluminescence in s.c. tumors. For the s.c. tumor model, atelocollagen was complexed with siRNA to a 'low' final concentration of atelocollagen $(0.05 \%)$, so that the siRNA/vector complexes presented a viscosity comparable to blood. SiRNAs - or vehicle - were injected i.v. at day 0,1 and 2, and bioluminescence was assessed at day 0 (before treatment), day 3 and 7, as described in Fig. 1B. An increase of bioluminescence over time was observed for $\mathrm{NaCl}$ and siGFP, consistent with the expected growth of tumors over time (Fig. 3A and B). However, at day 3 the bioluminescence values were significantly lower in siLuc compared to day 0 (47\% of day 0 ). This decrease was even stronger compared to the bioluminescence values of $\mathrm{NaCl}(319 \%$ of day 0$)$ with $\mathrm{p}=0.004$, and vs. $\operatorname{siGFP}(155 \%$ of day 0$)$ with $\mathrm{p}=0.015$ (Fig. 3C and D), which means a decrease in bioluminescence signal of 85 and $70 \%$ relative to $\mathrm{NaCl}$ and siGFP respectively. In contrast, there was no significant difference at day 7 between groups, with siLuc bioluminescence signal intensity catching up with other groups, supporting that the decrease in bioluminescence intensity observed at day 3 was not the consequence of impaired tumor growth and/or tumor mass reduction for siLuc group (Fig. 3 and data not shown).

Unlike luciferase-directed siRNA, Mcl-1- and Bcl- $x_{L}$-directed siRNAs vectorized with atelocollagen do not induce target downregulation in vivo. After defining the successful conditions for in vivo siRNA vectorization with atelocollagen, we sought to downregulate the cancer-related targets of interest, Mcl-1 and Bcl- $\mathrm{x}_{\mathrm{L}}$. We used siRNA sequences which had been validated by our group in previous studies for their ability to downregulate their targets specifically with high efficiency $(3,4,6)$. In addition, the batches of siMcl1 and siBcl- $\mathrm{x}_{\mathrm{L}}$ that we used in vivo were checked for their ability to downregulate their respective targets in vitro in SKOV3 cells. The complete disappearance of the protein-specific band was observed in western blotting (Fig. 4A), demonstrating that these siRNAs enable full silencing of their respective targets. These results were comparable to what was obtained upon siLuc transfection in SKOV3-Luc cells, which triggered
$89 \%$ luminescence inhibition compared to the control conditions (Fig. 4B).

SKOV3 cells were injected into nude mice to develop bilateral s.c. tumors and the above-described protocol and treatment schedule (Fig. 1B) were repeated, with 3 exceptions; i) siLuc condition was removed and replaced with siMcl1 or siBcl- $\mathrm{x}_{\mathrm{L}}$; ii) we discarded the day 7 analysis time point as it did not lead to a decrease in bioluminescence in the above-described experiment; and iii) we used 5 mice/group with a total of 20 mice. Unfortunately, neither RT-qPCR analysis performed on tumor lysates nor IHC performed in situ on tumor slices revealed any $\mathrm{Mcl}-1$ or $\mathrm{Bcl}-\mathrm{x}_{\mathrm{L}}$ downregulation at day 3 after the beginning of the protocol (Fig. 5).

\section{Discussion}

Our group has previously shown that the concomitant inhibition of Bcl- $\mathrm{x}_{\mathrm{L}}$ and $\mathrm{Mcl}-1$ is sufficient to trigger apoptosis in ovarian carcinoma cell lines (3), as well as in cell lines from other malignancies (37-39). The use of siRNAs is an attractive option to downregulate Bcl-2 anti-apoptotic family members in ovarian tumors, particularly Mcl-1 for which no pharmacological inhibitor is yet available for clinical use.

The development of safe and efficient tools for siRNA vectorization is a very intensive research topic. More than 50 clinical trials involving siRNAs have been reported (40) but no siRNA-based drug has yet obtained FDA approval and none of the vectorization systems utilized have achieved a consensus $(40,41)$. Furthermore, a large number of different vectorization approaches have been considered for in vivo preclinical studies $(40,42,43)$. The choice of a suitable system to vectorize siRNAs in vivo is still greatly dependent on the experimental model.

Ovarian carcinoma development can be classified into 4 different tumoral tissue types: primary tumor, peritoneal tumor including micro- and macro-carcinomatosis, distant tumor nodes, and ascites. The primary tumor is usually removed surgically during first-line treatment, and ascites in vivo in mice is usually present at a very late stage of tumor development so it cannot be studied for ethical reasons. 
A

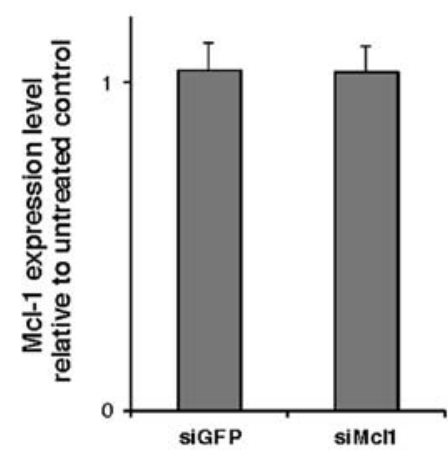

C

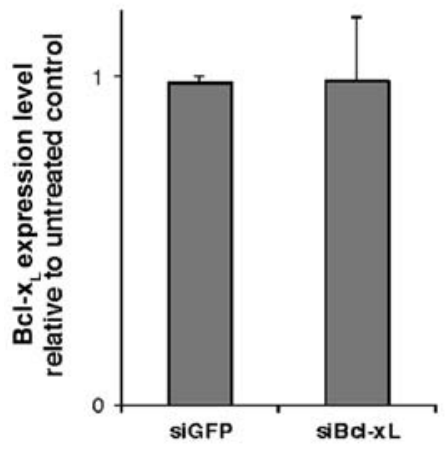

B

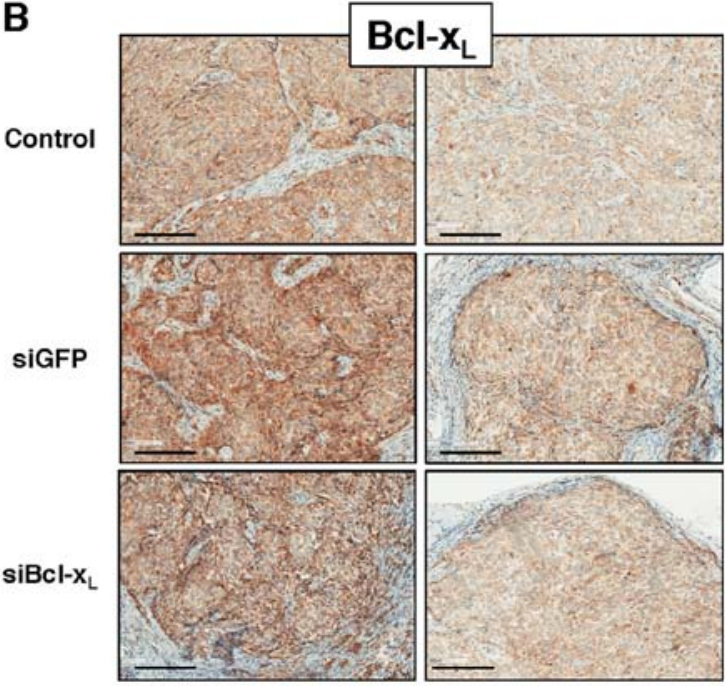

D

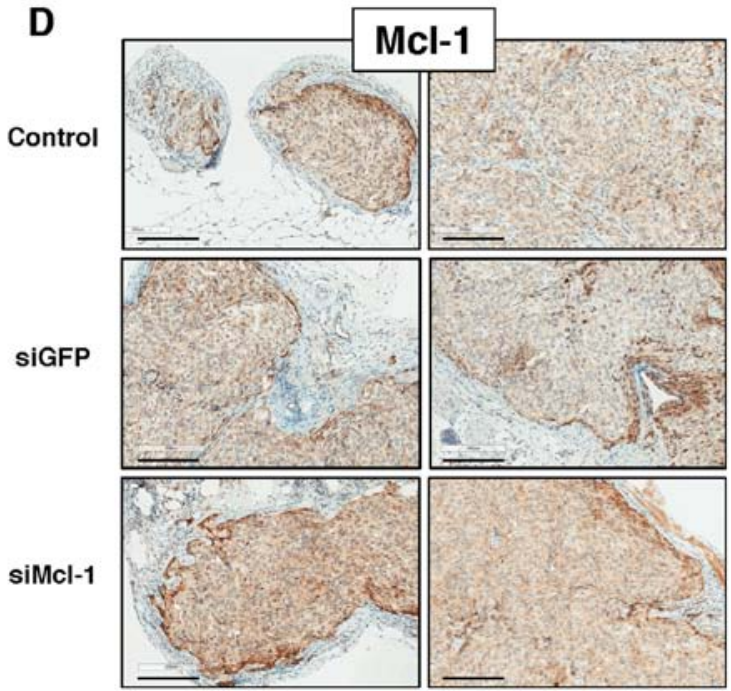

Figure 5. i.v. injection for Mcl-1 or Bcl- $\mathrm{x}_{\mathrm{L}}$-targeted siRNA to subcutaneous tumors. (A and C) RT-qPCR analysis of Mcl-1 and Bcl- $\mathrm{x}_{\mathrm{L}} \mathrm{mRNA}$ expression level in tumor nodes harvested at day 3. Expression is relative to $\mathrm{NaCl}$ tumors for siGFP, siMcl-1 or siBcl-xL. Data is presented as mean values from 5 tumor nodes from 5 different animals for siMcl-1 and 4 tumor nodes from 4 different animals for siBcl-xL, \pm SD. (B and D) IHC for Mcl-1 or Bcl- $\mathrm{x}_{\mathrm{L}}$ on tumor nodes harvested at day 3 . Images are representative of 6 different tumors in each group. Scale bar, $200 \mu \mathrm{m}$.

Micro-carcinomatosis and secondary tumor nodes, which are easily developed in mice, are a good model of the clinically challenging residual disease and recurrence so we targeted them in our siRNA experiments.

The aim of the present study was to evaluate the possibility to vectorize siRNAs in two in vivo models of tumoral development in mice mimicking the setting of ovarian carcinoma: the development of carcinomatosis matching the peritoneal dissemination mainly displaying small-sized tumor nodes with low vascularization, and subcutaneous tumors matching distant metastasis with larger tumor nodes displaying more developed vascularization. For the carcinomatosis model with its small islets of tumor cells lacking vascularization, local i.p. injection is attractive. For the distant and/or vascularized metastasis model with i.v. injection, s.c. tumor development is more relevant owing to the unique presence of larger vascularized tumor nodes. We chose to use atelocollagen as a vector since its flexibility (two possible formulations adapted to i.v. and i.p. administration, respectively) enabled us to use it in both models. In addition, the possibility to use each tumor as its own control, thereby avoiding inter-animal and internodular variability, led us to use a bioluminescent tumoral model to establish a suitable administration regimen.

In the peritoneal carcinomatosis model, we could not evidence any effect of atelocollagen-vectorized siRNAs on the bioluminescence signal in comparison to the control groups. To the best of our knowledge, this formulation with high viscosity has been used only for siRNA delivery with peritumoral injection $(18,21,22,30,31)$, with two exceptions. One study reported atelocollagen-vectorized siRNAs with i.p. injection for intraperitoneal NSCLC tumors (33), but the authors did not give any information either on the atelocollagen concentration or on the amount of siRNA used. Another study demonstrated efficient siRNA delivery and target downregulation on peritoneal tumor nodes in a gastric cancer model after injection of siRNAs-vectorized with atelocollagen at $0.5 \%$ together with DharmaFECT 1 transfection reagent (34). Notably, the use of atelocollagen 
alone in their model did not allow target downregulation, in accordance with our observations. However, the authors did not report the effects of DharmaFECT 1 transfection reagent in the absence of atelocollagen, so the contribution of atelocollagen to the successful vectorization of siRNAs and silencing of their target could not be estimated.

One of the reasons for the lack of efficacy in this model could be that, unlike with peritumoral or intratumoral injections, the complexes released in the peritoneal cavity are not maintained in contact with the majority of tumor cells, as they cannot cover the entirety of the peritoneal cavity. However, i.p. injection of atelocollagen complexed with ASO was used for delivery to s.c. tumors (44), indicating that small oligonucleotides are at least released efficiently in the peritoneal cavity even with an atelocollagen concentration of $1.8 \%$, which is higher than the one used in the present study, i.e. $0.5 \%$, the concentration recommended in the commercially available kit. A possibility is that once released in the peritoneal cavity, siRNAs, which are more prone to degradation than phosphorothioate-modified ASO, are rapidly excreted and/or degraded without the help of a complementary vector system, before they can reach tumor cells present in this compartment. In order to monitor biodistribution, direct labeling of siRNAs has been used in several instances in vivo. However, the peritoneal compartment is not an organ per se, and such an approach would be difficult to use in our case to monitor the intra-peritoneal release of siRNAs.

Regarding the s.c. tumor model, we first evidenced satisfactory efficiency on the inhibition of luciferase expression as revealed by in vivo bioluminescence (70\% decrease in signal vs. control siRNA), which is in line with previous studies in which siRNAs-vectorized with atelocollagen were used $(17,23,24,35)$ on tumors of various origins. This downregulation effect was present at day 3 after 3 daily consecutive injections and was absent at day 7 , showing that the effect is transient over time suggesting a loss of the RNA inhibition effect soon after the end of the injections. In several studies describing a long-lasting effect on target inhibition, the protocols included multiple repeated injections $(34,45)$, suggesting that without this, no long-lasting target inhibition can be obtained, which is again in line with our own observations. In addition, when targeting genes involved in cancer progression, successful inhibition by siRNAs prevents further tumoral development and enables tumoral regression. In our luciferase model, however, target inhibition is not supposed to, and indeed does not, impair tumor development. Therefore, the inhibitory effects of transfected siRNAs are likely to be diluted over cell divisions, which is another plausible explanation for a transient decrease in bioluminescence.

Unfortunately, we could not observe the inhibition of target gene expression in s.c. tumors using Mcl-1- or Bcl- $\mathrm{x}_{\mathrm{L}}$-directed siRNAs. The histology of tumors derived from SKOV3 cells (used during Mcl-1 and Bcl- $\mathrm{x}_{\mathrm{L}}$ experiments) or SKOV3-Luc cells (used during the bioluminescence experiments) are similar, as confirmed by a certified pathologist (data not shown). Therefore, the use of SKOV3 instead of SKOV3-Luc cells to generate tumors does not likely explain the differences observed between our in vivo experiments.

It has been shown that target mRNA half-life influences RNAi efficiency; mRNAs with shorter half-life being more difficult to downregulate (46). Luciferase, Mcl-1 and Bcl- $\mathrm{x}_{\mathrm{L}}$ mRNA half-lives are 1.5, 2.5 and $2-3 \mathrm{~h}$, respectively $(47,48)$. These small differences regarding mRNA half-lives are thus quite unlikely to induce any difference in the timing of mRNA downregulation following siRNA injection and do not constitute an explanation for the absence of observed $\mathrm{Bcl}-\mathrm{x}_{\mathrm{L}}$ or Mcl-1 downregulation. With regard to protein half-lives, it should be noted that luciferase and Mcl-1 proteins have a 6 -fold difference in their respective half-lives [3 h vs. $30 \mathrm{~min}$, respectively (49)]. In addition, the half-life of Bcl-xL protein has been reported to be $\sim 20 \mathrm{~h}$ (50). Due to these differences, the effects of siRNA downregulation on Mcl-1 protein should have been measurable earlier than luciferase, whereas Bcl- $\mathrm{x}_{\mathrm{L}}$ protein downregulation should have lasted longer. Overall, the time window in which we should have been able to observe any protein downregulation was larger with $\mathrm{Bcl}-\mathrm{x}_{\mathrm{L}}$ and $\mathrm{Mcl}-1$. Therefore any difference in the timing of the action of $\mathrm{Bcl}-\mathrm{x}_{\mathrm{L}}$ or Mcl-1 siRNAs compared to luciferase would have not compromised our ability to observe the effects of siRNA on protein, at least on one of our targets. In fact, these differences in the half-lives of our proteins of interest do not likely explain the lack of observed siRNA activity.

We did consider an increase in dose and/or number of administrations to obtain a more stable and reproducible target downregulation, as well as the use of a panel of alternative siRNA sequences for Mcl-1 and Bcl- $\mathrm{X}_{\mathrm{L}}$. However, in most of the animals we observed a seizure-like behavior shortly after i.v. injection of atelocollagen in complex with each of the 4 siRNAs we used. The mice recovered quickly with no visible sign of physiological or behavioral consequence. It is unlikely that this effect was triggered by contamination of our siRNAs as seizures were observable with 4 different PAGE-purified siRNAs. To the best of our knowledge, this effect with the use of atelocollagen has not been reported elsewhere. Considering previous studies in the literature and the manufacturer's protocol, we could not increase the siRNA concentration in the siRNA/vector complexes. Therefore, as we ruled out increasing the volume or frequency of injections for ethical reasons, we did not push our investigations further with atelocollagen for in vivo siRNA delivery.

In summary, formulation with a high concentration of atelocollagen alone is not suitable for i.p. siRNA transfection in peritoneal nodes. In contrast, a low concentration formulation for i.v. injection is able to deliver siRNAs in s.c. tumors and to induce a strong but transient silencing of the targeted protein activity. In addition, the efficiency of target silencing is very sensitive and depends on the nature of the target. Given that we used the limiting dose for this vector and that the level and duration of silencing are clearly insufficient to explore possible therapeutic effects, we decided not to push our investigations with this vector further for the delivery of siRNAs to ovarian cancer cell xenografts in vivo in mice.

\section{Acknowledgements}

The authors wish to thank Edwige Lemoisson for technical assistance, the CMAbio3 platform (SF 4206 ICORE UNICAEN) for IHC and the OPTIMAL platform (Grenoble) for small animal bioluminescence imaging. The present study is part of the national program Cartes d'Identite des 
Tumeurs (CIT) funded and developed by the Ligue Nationale Contre le Cancer. It was supported by the 'Ligue contre le Cancer' (Calvados and Orne Committee), the 'Conseil Regional de Basse-Normandie', and the French Government. E.B., M.M.F., E.V. and C.L. were recipients of a doctoral fellowship from the 'Ligue contre le Cancer' (Calvados Committee: E.B., E.V. and C.L.; Eure Committee: M.M.F.) and C.D. was recipient of a post-doctoral fellowship from the 'Conseil Regional de Basse-Normandie'. N.V. was a recipient of a doctoral fellowship from the French Ministry for Higher Education and Research. The present study was supported by a grant from l'Ecole de l'Inserm Liliane Bettencourt (N.V.).

\section{References}

1. Villedieu M, Louis MH, Dutoit S, Brotin E, Lincet H, Duigou F, Staedel C, Gauduchon P and Poulain L: Absence of Bcl- $x_{L}$ down-regulation in response to cisplatin is associated with chemoresistance in ovarian carcinoma cells. Gynecol Oncol 105: 31-44, 2007.

2. Tomasina J, Malzert-Freon A, Giffard F, Brotin E, Louis MH, Abeilard E, Rault S, Gauduchon P and Poulain L: Sensitization of ovarian carcinoma cells to $\mathrm{Bcl}-\mathrm{x}_{\mathrm{L}}$-targeting strategies through indirect modulation of Mcl-1 activity by MR22388, a molecule of the tripentone family. J Ovarian Res 6: 38-2013, 2013.

3. Brotin E, Meryet-Figuière M, Simonin K, Duval RE, Villedieu M, Leroy-Dudal J, Saison-Behmoaras E, Gauduchon P, Denoyelle C and Poulain L: Bcl- $\mathrm{X}_{\mathrm{L}}$ and MCL-1 constitute pertinent targets in ovarian carcinoma and their concomitant inhibition is sufficient to induce apoptosis. Int J Cancer 126: 885-895, 2010.

4. Varin E, Denoyelle C, Brotin E, Meryet-Figuière M, Giffard F, Abeilard E, Goux D, Gauduchon P, Icard P and Poulain L: Downregulation of $\mathrm{Bcl}-\mathrm{xL}$ and $\mathrm{Mcl}-1$ is sufficient to induce cell death in mesothelioma cells highly refractory to conventional chemotherapy. Carcinogenesis 31: 984-993, 2010.

5. Simonin K, Brotin E, Dufort S, Dutoit S, Goux D, N'diaye M, Denoyelle C, Gauduchon P and Poulain L: Mcl-1 is an important determinant of the apoptotic response to the $\mathrm{BH} 3$-mimetic molecule HA14-1 in cisplatin-resistant ovarian carcinoma cells. Mol Cancer Ther 8: 3162-3170, 2009.

6. Simonin K, N'Diaye M, Lheureux S, Loussouarn C, Dutoit S, Briand M, Giffard F, Brotin E, Blanc-Fournier C and Poulain L: Platinum compounds sensitize ovarian carcinoma cells to ABT-737 by modulation of the Mcl-1/Noxa axis. Apoptosis 18: 492-508, 2013.

7. Lheureux S, N'diaye M, Blanc-Fournier C, Dugue AE, Clarisse B, Dutoit S, Giffard F, Abeilard E, Briand M, Labiche A, et al: Identification of predictive factors of response to the BH3-mimetic molecule ABT-737: An ex vivo experiment in human serous ovarian carcinoma. Int J Cancer 136, E340-E350, 2015.

8. Gloaguen C, Voisin-Chiret AS, Sopkova-de Oliveira Santos J, Fogha J, Gautier F, De Giorgi M, Burzicki G, Perato S, Pétigny-Lechartier C, Simonin-Le Jeune K, et al: First evidence that oligopyridines, $\alpha$-helix foldamers, inhibit Mcl- 1 and sensitize ovarian carcinoma cells to Bcl-xL-targeting strategies. J Med Chem 58: 1644-1668, 2015.

9. Letai AG: Diagnosing and exploiting cancer's addiction to blocks in apoptosis. Nat Rev Cancer 8: 121-132, 2008.

10. Certo M, Del Gaizo Moore V, Nishino M, Wei G, Korsmeyer S, Armstrong SA and Letai A: Mitochondria primed by death signals determine cellular addiction to antiapoptotic BCL-2 family members. Cancer Cell 9: 351-365, 2006.

11. Belmar J and Fesik SW: Small molecule Mcl-1 inhibitors for the treatment of cancer. Pharmacol Ther 145: 76-84, 2015.

12. Varadarajan S, Vogler M, Butterworth M, Dinsdale D, Walensky LD and Cohen GM: Evaluation and critical assessment of putative MCL-1 inhibitors. Cell Death Differ 20: 1475-1484, 2013.

13. Leverson JD, Zhang H, Chen J, Tahir SK, Phillips DC, Xue J, Nimmer P, Jin S, Smith M, Xiao Y, et al: Potent and selective small-molecule MCL-1 inhibitors demonstrate on-target cancer cell killing activity as single agents and in combination with ABT-263 (navitoclax). Cell Death Dis 6: e1590, 2015.
14. Deleavey GF and Damha MJ: Designing chemically modified oligonucleotides for targeted gene silencing. Chem Biol 19: 937-954, 2012.

15. Colombo S, Zeng X, Ragelle H and Foged C: Complexity in the therapeutic delivery of RNAi medicines: An analytical challenge. Expert Opin Drug Deliv 11: 1481-1495, 2014.

16. Miller AD: Delivery of RNAi therapeutics: Work in progress. Expert Rev Med Devices 10: 781-811, 2013.

17. Takeshita F, Minakuchi Y, Nagahara S, Honma K, Sasaki H, Hirai K, Teratani T, Namatame N, Yamamoto Y, Hanai K, et al: Efficient delivery of small interfering RNA to bone-metastatic tumors by using atelocollagen in vivo. Proc Natl Acad Sci USA 102: 12177-12182, 2005

18. Takei Y, Kadomatsu K, Yuzawa Y, Matsuo S and Muramatsu T: A small interfering RNA targeting vascular endothelial growth factor as cancer therapeutics. Cancer Res 64: 3365-3370, 2004.

19. Fujimoto I and Takei Y: Atelocollagen-mediated siRNA delivery: Future promise for therapeutic application. Ther Deliv 5: 369-371, 2014.

20. Svintradze DV and Mrevlishvili GM: Fiber molecular model of atelocollagen-small interfering RNA (siRNA) complex. Int J Biol Macromol 37: 283-286, 2005.

21. Ochiya T, Nagahara S, Sano A, Itoh $\mathrm{H}$ and Terada M: Biomaterials for gene delivery: atelocollagen-mediated controlled release of molecular medicines. Curr Gene Ther 1: 31-52, 2001.

22. Forootan SS, Bao ZZ, Forootan FS, Kamalian L, Zhang Y, Bee A, Foster CS and Ke Y: Atelocollagen-delivered siRNA targeting the FABP5 gene as an experimental therapy for prostate cancer in mouse xenografts. Int J Oncol 36: 69-76, 2010.

23. Kawata E, Ashihara E, Kimura S, Takenaka K, Sato K, Tanaka R, Yokota A, Kamitsuji Y, Takeuchi M, Kuroda J, et al: Administration of PLK-1 small interfering RNA with atelocollagen prevents the growth of liver metastases of lung cancer. Mol Cancer Ther 7: 2904-2912, 2008.

24. Mu P, Nagahara S, Makita N, Tarumi Y, Kadomatsu K and Takei Y: Systemic delivery of siRNA specific to tumor mediated by atelocollagen: Combined therapy using siRNA targeting Bcl-xL and cisplatin against prostate cancer. Int J Cancer 125: 2978-2990, 2009.

25. Minakuchi Y, Takeshita F, Kosaka N, Sasaki H, Yamamoto Y, Kouno M, Honma K, Nagahara S, Hanai K, Sano A, et al: atelocollagen-mediated synthetic small interfering RNA delivery for effective gene silencing in vitro and in vivo. Nucleic Acids Res 32: e109-e2004, 2004.

26. Nozawa H, Tadakuma T, Ono T, Sato M, Hiroi S, Masumoto K and Sato Y: Small interfering RNA targeting epidermal growth factor receptor enhances chemosensitivity to cisplatin, 5-fluorouracil and docetaxel in head and neck squamous cell carcinoma. Cancer Sci 97: 1115-1124, 2006.

27. Iwaki K, Shibata K, Ohta M, Endo Y, Uchida H, Tominaga M, Okunaga R, Kai S and Kitano S: A small interfering RNA targeting proteinase-activated receptor- 2 is effective in suppression of tumor growth in a Pancl xenograft model. Int J Cancer 122: 658-663, 2008

28. Takei Y, Kadomatsu K, Goto T and Muramatsu T: Combinational antitumor effect of siRNA against midkine and paclitaxel on growth of human prostate cancer xenografts. Cancer 107: 864-873, 2006.

29. Takigami I, Ohno T, Kitade Y, Hara A, Nagano A, Kawai G, Saitou M, Matsuhashi A, Yamada K and Shimizu K: Synthetic siRNA targeting the breakpoint of EWS/Fli-1 inhibits growth of Ewing sarcoma xenografts in a mouse model. Int J Cancer 128: 216-226, 2011.

30. Ashihara E, Kawata E, Nakagawa Y, Shimazaski C, Kuroda J, Taniguchi K, Uchiyama H, Tanaka R, Yokota A, Takeuchi M, et al: $\beta$-Catenin small interfering RNA successfully suppressed progression of multiple myeloma in a mouse model. Clin Cancer Res 15: 2731-2738, 2009.

31. Sudo H, Tsuji AB, Sugyo A, Kohda M, Sogawa C, Yoshida C, Harada YN, Hino O and Saga T: Knockdown of COPA, identified by loss-of-function screen, induces apoptosis and suppresses tumor growth in mesothelioma mouse model. Genomics 95: 210-216, 2010.

32. Koyanagi T, Suzuki Y, Saga Y, Machida S, Takei Y, Fujiwara H, Suzuki M and Sato Y: In vivo delivery of siRNA targeting vasohibin-2 decreases tumor angiogenesis and suppresses tumor growth in ovarian cancer. Cancer Sci 104: 1705-1710, 2013. 
33. Tasaki M, Shimada K, Kimura H, Tsujikawa K and Konishi N ALKBH3, a human AlkB homologue, contributes to cell survival in human non-small-cell lung cancer. Br J Cancer 104: 700-706, 2011.

34. Fujita T, Yanagihara K, Takeshita F, Aoyagi K, Nishimura T, Takigahira M, Chiwaki F, Fukagawa T, Katai H, Ochiya T, et al: Intraperitoneal delivery of a small interfering RNA targeting NEDD1 prolongs the survival of scirrhous gastric cancer model mice. Cancer Sci 104: 214-222, 2013.

35. Azuma K, Nakashiro K, Sasaki T, Goda H, Onodera J, Tanji N, Yokoyama $\mathrm{M}$ and Hamakawa $\mathrm{H}$ : Anti-tumor effect of small interfering RNA targeting the androgen receptor in human androgen-independent prostate cancer cells. Biochem Biophys Res Commun 391: 1075-1079, 2010.

36. Sasaki T, Nakashiro K, Tanaka H, Azuma K, Goda H, Hara S, Onodera J, Fujimoto I, Tanji N, Yokoyama M, et al: Knockdown of Akt isoforms by RNA silencing suppresses the growth of human prostate cancer cells in vitro and in vivo. Biochem Biophys Res Commun 399: 79-83, 2010.

37. Woo SM, Min KJ, Seo BR, Nam JO, Choi KS, Yoo YH, and Kwon TK: Cafestol overcomes ABT-737 resistance in Mcl-1-overexpressed renal carcinoma Caki cells through downregulation of Mcl-1 expression and upregulation of Bim expression. Cell Death Dis 5: e1514, 2014.

38. Sadahira K, Sagawa M, Nakazato T, Uchida H, Ikeda Y, Okamoto S, Nakajima $\mathrm{H}$ and Kizaki M: Gossypol induces apoptosis in multiple myeloma cells by inhibition of interleukin- 6 signaling and Bcl-2/Mcl-1 pathway. Int J Oncol 45: 2278-2286, 2014.

39. Cao X, Yap JL, Newell-Rogers MK, Peddaboina C, Jiang W, Papaconstantinou HT, Jupitor D, Rai A, Jung KY, Tubin RP, et al: The novel BH3 $\alpha$-helix mimetic JY-1-106 induces apoptosis in a subset of cancer cells (lung cancer, colon cancer and mesothelioma) by disrupting $\mathrm{Bcl}-\mathrm{xL}$ and $\mathrm{Mcl}-1$ protein-protein interactions with Bak. Mol Cancer 12: 42-2013, 2013.

40. Ozcan G, Ozpolat B, Coleman RL, Sood AK and LopezBerestein G: Preclinical and clinical development of siRNA-based therapeutics. Adv Drug Deliv Rev 87: 108-119, 2015.
41. Wu SY, Lopez-Berestein G, Calin GA and Sood AK: RNAi therapies: Drugging the undruggable. Sci Transl Med 6: 240ps7, 2014.

42. Guo J, Fisher KA, Darcy R, Cryan JF and O'Driscoll C: Therapeutic targeting in the silent era: Advances in non-viral siRNA delivery. Mol Biosyst 6: 1143-1161, 2010.

43. Kanasty R, Dorkin JR, Vegas A and Anderson D: Delivery materials for siRNA therapeutics. Nat Mater 12: 967-977, 2013.

44. Nakazawa K, Nemoto T, Hata T, Seyama Y, Nagahara S, Sano A, Itoh H, Nagai Y and Kubota S: Single-injection ornithine decarboxylase-directed antisense therapy using atelocollagen to suppress human cancer growth. Cancer 109: 993-1002, 2007.

45. Bosma GC, Custer RP and Bosma MJ: A severe combined immunodeficiency mutation in the mouse. Nature 301: 527-530, 1983.

46. Larsson E, Sander C, and Marks D: mRNA turnover rate limits siRNA and microRNA efficacy. Mol Syst Biol 6: 433, 2010.

47. Wilsbacher LD, Yamazaki S, Herzog ED, Song EJ, Radcliffe LA, Abe M, Block G, Spitznagel E, Menaker M and Takahashi JS: Photic and circadian expression of luciferase in mPeriod1-luc transgenic mice invivo. Proc Natl Acad Sci USA 99: 489-494, 2002.

48. Reed JC, Tsujimoto Y, Epstein SF, Cuddy M, Slabiak T, Nowell PC and Croce CM: Regulation of bcl-2 gene expression in lymphoid cell lines containing normal \#18 or $\mathrm{t}(14 ; 18)$ chromosomes. Oncogene Res 4: 271-282, 1989.

49. Wei G, Margolin AA, Haery L, Brown E, Cucolo L, Julian B, Shehata S, Kung AL, Beroukhim R and Golub TR: Chemical genomics identifies small-molecule MCL1 repressors and BCL-xL as a predictor of MCL1 dependency. Cancer Cell 21: $547-562,2012$.

50. Rooswinkel RW, van de Kooij B, de Vries E, Paauwe M, Braster R, Verheij M and Borst J: Antiapoptotic potency of Bcl-2 proteins primarily relies on their stability, not binding selectivity. Blood 123: 2806-2815, 2014. 